\title{
AUTHOR INDEX \\ VOLUME 10 (2002)
}

Agostini, V., see Scalerandi, M.

10, 3 (2002) 275-294

Ali, A.A., see Atassi, O.V.

Atassi, O.V. and Ali, A.A., Inflow/outflow conditions for time-harmonic internal flows

Avital, E.J., Optimized differentiation schemes on non-uniform grids for computational aeroacoustics

10, 2 (2002) 155-182

10, 2 (2002) 155-182

Broschat, S.L., see Zhang, X.

Buckingham, M.J., Giddens, E.M., Simonet, F., and Hahn, T.R., Propeller noise from a light aircraft for low-frequency measurements of the speed of sound in a marine sediment

Dong, Y., see Du, Q.

Dosso, S.E., Benchmarking range-dependent propagation modeling in matched-field inversion

Du, Q., Yang, H.-Z., and Dong, Y., A study on shear wave velocity estimation and fracture detection in HTI media

Dyson, R.W., Technique for very high order nonlinear simulation and validation

10, 2 (2002) 195-209

10, 2 (2002) 243-264

10, 4 (2002) 445-464

10, 3 (2002) 331-347

10, 2 (2002) 231-242

10, 3 (2002) 331-347

10, 2 (2002) 211-229

10, 2 (2002) 243-264

Flynn, P.J., see Zhang, X.

Franco, F.G., Godinho, L.M.C., and Tadeu, A.J.B., Propagation of pressure waves inside a confined fluid channel with an irregular floor

10, 2 (2002) 183-194

Fung, K.-Y., see Ju, H.B.

Garcia, J., see George, A.D.

10, 3 (2002) 311-329

10, 1 (2002) 1-23

George, A.D., see Sinha, P.

10, 1 (2002) 69-96

George, A.D., Garcia, J., Kim, K., and Sinha, P., Distributed parallel processing techniques for adaptive sonar beamforming

Giddens, E.M., see Buckingham, M.J.

Godin, O.A., A 2-D description of sound propagation in a horizontally-inhomogeneous ocean

10, 1 (2002) 1-23

10, 4 (2002) 445-464

Godinho, L.M.C., see Franco, F.G.

10, 1 (2002) 123-151

10, 2 (2002) 183-194

Hahn, T.R., see Buckingham, M.J.

Ju, H.B. and Fung, K.-Y., Time-domain simulation of acoustic sources over an impedance plane

10, 4 (2002) 445-464

10, 3 (2002) 311-329

Kampanis, N.A., Numerical simulation of low-frequency aeroacoustics over irregular terrain using a finite element discretization of the parabolic equation

10, 1 (2002) 97-111 
Kim, J.-H., Refraction and diffusion of acoustic waves in a random fluid medium

10, 2 (2002) 265-274

Kim, K., see George, A.D.

Kim, K., see Sinha, P.

Lee, D., see Nagem, R.J.

Lee, D., Computational atmospheric acoustics, by E. M. Salomons

Li, L.-X., Sun, J.-S., and Sakamoto, H., A modified infinite element method for acoustic radiation

Marburg, S., Six boundary elements per wavelength: Is that enough?

Mariani, F., Recchioni, M.C., and Zirilli, F., A perturbative approach to acoustic scattering from a vibrating bounded obstacle

McDaniel, S.T., Renormalizing rough-surface scatter

Nagem, R.J. and Lee, D., Coupled 3D wave equations with irregular fluid-elastic interface: Theoretical development

Recchioni, M.C., see Mariani, F.

Sakamoto, H., see Li, L.-X.

Scalerandi, M. and Agostini, V., Simulation of the propagation of ultrasonic pulses in nonlinear and/or attenuative media

Simonet, F., see Buckingham, M.J.

Sinha, P., see George, A.D.

Sinha, P., George, A.D., and Kim, K., Parallel algorithms for robust broadband MVDR beamforming

Smith, K.B., Computing the influence of doppler due to source/receiver motion in a parabolic equation model

Sun, J.-S., see Li, L.-X.

Tadeu, A.J.B., see Franco, F.G.

Tam, C.K.W., Computational aeroacoustics examples showing the failure of the acoustic analogy theory to identify the correct noise sources

Williams, J.E.F., Address given at David Crighton's memorial service, June 3, 2000

Wu, S.F., Absolute instability or chaos? - A tribute to Dr. David G. Crighton

Yang, H.-Z., see Du, Q.

Zhang, X., Broschat, S.L., and Flynn, P.J., A conjugate gradient-neural network technique for ultrasound inverse imaging Zirilli, F., see Mariani, F.

10, 1 (2002) 1-23

10, 1 (2002) 69-96

10, 4 (2002) 421-444

10, 1 (2002) 153-154

10, 1 (2002) 113-121

10, 1 (2002) 25-51

10, 3 (2002) 349-384

10, 1 (2002) 53-68

10, 4 (2002) 421-444

10, 3 (2002) 349-384

10, 1 (2002) 113-121

10, 3 (2002) 275-294

10, 4 (2002) 445-464

10, 1 (2002) 1-23

10, 1 (2002) 69-96

10, 3 (2002) 295-309

10, 1 (2002) 113-121

10, 2 (2002) 183-194

10, 4 (2002) 387-405

10, 4 (2002) ix-xiii

10, 4 (2002) 407-419

10, 3 (2002) 331-347

10, 2 (2002) 243-264

10, 3 (2002) 349-384 